The prevalence of rectal gonorrhoea $(2.8 \%$ vs $3.5 \%, p=0.472)$, rectal chlamydia $(5.9 \%$ vs $6.8 \%, p=0.496)$ and early syphilis $(1.5 \%$ vs $2.2 \%, p=0.346)$ were similar in the two age groups. More teenage MSM had pharyngeal gonorrhoea $(4.8 \%$ vs $2.0 \%$, p < 0.001$)$ but more older MSM were diagnosed for urethral chlamydia $(0.7 \%$ vs $3.3 \%, p=0.004)$ and $\operatorname{HIV}(0.3 \%$ vs $1.8 \%, p=0.021)$.

Conclusion A high level of sexual risk was seen among teenage MSM together with a high prevalence of STIs. More innovative and age-specific measures should be adopted to promote sexual health messages to younger gay men.

\section{P3.161 TRIPLE-DIP: EXPANDED EXTRAGENITAL TESTING FOR NEISSERIA GONORRHOEAE AND CHLAMIDIA TRACHOMATIS IDENTIFIES HIGH RATES OF ASYMPTOMATIC INFECTION IN PERSONS LIVING WITH HIV}

doi:10.1136/sextrans-2013-051184.0619

M Sabo, H E L Reno, R Presti, B P Stoner. Washington University School of Medicine, Saint Louis, MO, United States

Background US guidelines now call for expanded extragenital testing for Neisseria gonorrhoeae (GC) and Chlamydia trachomatis (Ct) in HIV infected individuals. In January 2012, we instituted a new policy to promote routine three-site testing (genital, oropharyngeal, rectal) for GC/Ct among HIV-infected persons in our clinic population. The purpose of this study is to assess implementation of the "triple-dip" programme, as well as the prevalence and incidence of STI at each site.

Methods We conducted a retrospective chart review of HIVinfected patients seen in our clinic before (Jan.-Dec. 2011) and after (Jan.-Dec. 2012) implementation of a routine three-site testing policy, to compare GC/Ct prevalence during these two time periods. Self-reported behavioural data were also evaluated.

Results For the three months after the transitioning from symptom-triggered testing to routine three-site screening for $\mathrm{GC} / \mathrm{Ct}$, the number of oropharyngeal tests performed increased from 38 to 325 , and the number of rectal tests increased from 32 to 290, an 8 to 9 fold increase in testing. Although the rate of infection at most sites decreased with increased screening, the rate of rectal $\mathrm{GC} / \mathrm{Ct}$ remained unchanged (13\% pre-expanded testing verses $12 \%$ after initiating broader testing, $p=n$.s.). This suggests that the prevalence of asymptomatic rectal infections in patients living with HIV in our clinic is high. Preliminary analyses indicate that rectal infections are more common in our tested patient population $(12 \%)$ than at other sites of testing (4.5\% oropharyngeal tests were positive, $1.5 \%$ genital tests were positive).

Conclusion Although extragenital testing increased with expanded testing, not all patients at risk were screened. Given the higher percentage of positive rectal tests, enhanced testing should focus on increasing awareness of rectal infection, treatment intervention, and risk counselling.

\section{P3.162 AN ESTIMATE OF THE PROPORTION OF GONOCOCCAL, CHLAMYDIAL AND NON-GONOCOCCAL NON-CHLAMYDIAL URETHRITIS (NGNCU) ATTRIBUTABLE TO ORAL SEX AMONG MEN WHO HAVE SEX WITH MEN (MSM)}

doi:10.1136/sextrans-2013-051184.0620

L A Barbee, J C Dombrowski, L E Manhart, M R Golden. University of Washington, Seattle, WA, United States

Background The proportion of infectious urethritis associated with oral sex is unknown.

Methods We conducted a retrospective study of MSM diagnosed with symptomatic urethritis attending an STD Clinic between 2001-2010. We categorised men according to their urethral exposures in the previous 60 days: (1) only insertive oral sex and no insertive anal sex (IOS); (2) only protected insertive anal intercourse and insertive oral sex (PIAI); (3) unprotected insertive anal intercourse with or without oral sex (UIAI); (4) no insertive sex (oral or anal). We calculated the proportion of urethritis cases by groups as a minimum estimate of the proportion of cases attributable to oral sex.

Results Between 2001-2010, 4,091 MSM were diagnosed with urethritis, had complete records for categorization, and were included in this analysis. Men reported the following urethral exposures: $13 \%$ IOS, $21 \%$ PIAI, $65 \%$ UIAI, and $<1 \%$ no urethral exposure. Among 1,506 cases of gonococcal urethritis, $72 \%$ occurred among men reporting UIAI and $27.8 \%$ (95\% CI $25.5 \%-30.1 \%$ ) occurred in MSM reporting oral sex as their only urethral exposure (9.4\% IOS and $18.4 \%$ PIAI) in the last 60 days. Of the 787 cases of chlamydia urethritis, $71 \%$ were in men reporting UIAI, $8.8 \%$ IOS and $19.6 \%$ PIAI, making $28.3 \%$ (95\% CI $25.2 \%-31.6 \%$ ) of chlamydial urethritis cases attributable to oral exposure in the prior 60 days. Among 1,999 cases of NGNCU, UIAI accounted for $59 \%$ of cases; oral sex accounted for $43.1 \%$ (95\% CI $40.9 \%-45.3 \%$ ). $17 \%$ and $24 \%$ of NGNCU cases occurred in men reporting IOS and PIAI, respectively.

Conclusion While usually considered a safer sexual practise, our findings suggest that a large proportion of all cases of urethritis are attributable to insertive oral sex. These findings highlight the importance of screening the oropharynx and counselling MSM about the risks of oral sex.

\section{P3.163 HEPATITIS B AND HEPATITIS C VIRUS PREVALENCE AMONG SEXUALLY TRANSMITTED DISEASE PATIENTS IN FARWANIA REGION OF KUWAIT}

doi:10.1136/sextrans-2013-051184.0621

B S N Al-Mutairi. Farwania Hospital, Kuwait, Ardiya, Kuwait

Background HBV and HCV share similar modes of transmission including the sexual route. This study was conducted to determine the prevalence of HBV and HCV infections among STD patients in Farwaniya region of Kuwait.

Methods 1298 patients (1240 M, 58 F) presenting with history and/or signs and symptoms suggestive of an STD, seen over a period of one-year (January 2012 to December 2012) presenting to Farwania hospital dermatology department were included. Serology for HBV, HCV, HIV, and syphilis were done in all patients. HBV and HCV serology were performed in 1148 age and sex matched controls also, attending the same clinic with non-STD dermatological conditions.

Results Mean age + SD of patients was $33.91+9.70$ years (Age range: $19-58$ years). Majority of the patients were heterosexual (99.6\%). No history of blood transfusion, surgery, hospitalisation, parenteral drug use or traditional healing practises was found in any of the patients. Urethral discharge was the most common diagnosis (584), followed by genital warts (306), genital herpes (175), mollusca contagiosa (69) and syphilis (8). History of sexual encounter with concern/suspicion for an STD was reported by 166 patients. Most of the patients were expats. H/o recent travel was present in 159 patients. HCV was detected in $12 / 1298$ patients $(0.92 \%)$ and $6 / 1148$ controls $(0.52 \%)$. Serology for HBV and HIV were negative in all patients as well as controls.

Discussion Sexual transmission of HCV is low and controversial especially among monogamous heterosexuals. It is being recognised as an emerging STD among HIV positive homosexuals. Detection of HCV among more number of STD patients $(0.92 \%)$ without other risk factors, compared to non-STD dermatology patients $(0.52 \%)$ in Farwania, Kuwait emphasises that sexual transmission of HCV is possible. 